\title{
High Current Density 2D/3D Esaki Tunnel Diodes
}

Sriram Krishnamoorthy ${ }^{\dagger}{ }^{*}$, Edwin W. Lee II $I^{\dagger}$, Choong Hee Lee ${ }^{\dagger}$, Yuewei Zhang ${ }^{\dagger}$, William D. McCulloch ${ }^{\S}$, Jared M. Johnson", Jinwoo Hwang ${ }^{*}$, Yiying $\mathrm{Wu}^{\S}$, and Siddharth Rajan ${ }^{\text {t**}}$

${ }^{\dagger}$ Department of Electrical and Computer Engineering, ${ }^{*}$ Department of Materials Science and Engineering, and ${ }^{\S}$ Department of Chemistry and Biochemistry, The Ohio State University, Columbus, $\mathrm{OH} 43210$, United States

KEYWORDS

$\mathrm{MoS}_{2}$, tunnel diode, GaN, 2D/3D heterojunction, interband tunneling. ABSTRACT

The integration of two-dimensional materials such as transition metal dichalcogenides with bulk semiconductors offer interesting opportunities for 2D/3D heterojunction-based novel device structures without any constraints of lattice matching. By exploiting the favorable band alignment at the GaN/MoS 2 heterojunction, an Esaki interband tunnel diode is demonstrated by transferring large area, $\mathrm{Nb}$-doped, p-type $\mathrm{MoS}_{2}$ onto heavily n-doped GaN. A peak current density of $446 \mathrm{~A} / \mathrm{cm}^{2}$ with repeatable room temperature negative differential resistance, peak to valley current ratio of 1.2, and minimal hysteresis was measured in the $\mathrm{MoS}_{2} / \mathrm{GaN}$ non-epitaxial tunnel diode. A high current density of $1 \mathrm{kA} / \mathrm{cm}^{2}$ was measured in the Zener mode (reverse bias) at $-1 \mathrm{~V}$ bias. The $\mathrm{GaN} / \mathrm{MoS}_{2}$ tunnel junction was also modeled by treating $M_{0} S_{2}$ as a bulk semiconductor, and the electrostatics at the 


\section{D/3D interface was found to be crucial in explaining the experimentally observed device}

characteristics.

Two-dimensional (2D) layered materials such as transition metal dichalcogenides(TMDs) ${ }^{1}$ with no out-of-plane bonds offer interesting opportunities for integration with bulk semiconductors such as $\mathrm{Si}, \mathrm{GaAs}$ and $\mathrm{GaN}$. Integration of diverse bulk semiconductors is limited by the need for lattice matching and extended defect control. With no constraints of lattice matching, 2D materials can be integrated with bulk semiconductors to create novel vertical and lateral heterostructures. More recently, researchers have started exploring such 2D/3D heterojunctions for device applications. $\mathrm{MoS}_{2} / \mathrm{Si}$ heterojunctions have been investigated for device applications such as photodetectors ${ }^{2,3,4}$ and solar cells ${ }^{5}$. Researchers have also studied $\mathrm{MoS}_{2} / \mathrm{GaAs}^{6,7}, \mathrm{MoS}_{2} / \mathrm{InP}^{8}, \mathrm{MoS}_{2} / \mathrm{SiC}^{9}$ and $\mathrm{MoS}_{2} / \mathrm{GaN}^{10,11,12,13}$ heterojunctions for a range of device applications . 2D/2D $\left(\mathrm{MoS}_{2} / \mathrm{WSe}_{2}{ }^{14,15,16}, \mathrm{BP} / \mathrm{SnSe}_{2}{ }^{17}, \mathrm{WSe}_{2} / \mathrm{SnSe}_{2}{ }^{18}\right)$ and 2D/3D $\left(\mathrm{MoS}_{2} / \mathrm{Ge}\right)^{19}$ heterojunctions-based interband tunnel diodes and tunneling field effect transistors have also been experimentally demonstrated. 2D/3D heterojunctions are in particular very attractive for low power logic devices with steep subthreshold slope ${ }^{19}$ as $2 \mathrm{D}$ channel material enables excellent electrostatic gate control, and the use of 3D semiconductor enables ultra-sharp doping profiles and engineering of the band lineup to increase tunneling current.

Integration of 2D-layered materials with wide band gap semiconductors such as $\mathrm{GaN}$ is particularly interesting due to the complimentary properties of these material systems. While $\mathrm{GaN}$ and $\mathrm{AlN}$ have high breakdown fields enabling high power/voltage device operation, there are fundamental challenges in p-type doping of $\mathrm{GaN}, \mathrm{AlN}^{20}$ and $\mathrm{ZnO}^{21}$. Transition metal dichalcogenides with lower band gap can offer excellent current spreading properties due to high mobility even when they are atomically thin. Previous investigations on GaN/MoS 2 PN diodes ${ }^{10}$ 
indicated a very low value of conduction band offset $\left(\Delta \mathrm{E}_{\mathrm{c}} \sim 0.2 \mathrm{eV}\right)$. The very small value of conduction band offset resulted in a space charge region in $\mathrm{GaN}$ with a built-in potential $\left(\mathrm{V}_{\mathrm{bi}}=\right.$ $1.5 \mathrm{~V})$ much smaller than the band gap of $\mathrm{GaN}(3.4 \mathrm{eV})^{10}$. Such a band line up is expected to be suitable for an Esaki interband tunnel diode ${ }^{22}$ provided the depletion width is designed to be sufficiently narrow by employing heavy doping. In this work, we report a $\mathrm{p}^{+} \mathrm{MoS}_{2} / \mathrm{n}^{+} \mathrm{GaN}$ interband tunnel diode formed by transferring multilayer $\mathrm{Nb}$-doped $\mathrm{p}^{+} \mathrm{MoS}_{2}$ to molecular beam epitaxy grown $\mathrm{n}^{+} \mathrm{GaN}$. We observe high forward and reverse tunneling current with the characteristic negative differential resistance observed in forward bias at room temperature. Minimal hysteresis measured in the IV characteristics indicates the promise of such non-epitaxial heterointerfaces for extreme band engineering without lattice matching constraints.

$\mathrm{p}^{+} \mathrm{MoS}_{2}$ was grown by sulfurizing a sputtered Mo/Nb/Mo stack on sapphire ( $1 \mathrm{~cm}$ x 0.8 cm) with $\mathrm{MoS}_{2}$ powder as the sulfur source in a vacuum sealed quartz tube at $1100^{\circ} \mathrm{C} . \mathrm{Nb}$ acts as p-type dopant in $\mathrm{MoS}_{2}{ }^{23,24}$ and we have reported large area heavily p-type doped multilayer $\mathrm{MoS}_{2}$ on sapphire ${ }^{25}$ using this approach ${ }^{26}$. Large-area multilayer, p-type $\mathrm{MoS}_{2}$ was characterized by X-ray diffraction(XRD) and the films were found to be crystalline as evident from (002), (004) and (006) $\mathrm{MoS}_{2}$ peaks observed in XRD spectra (Figure 1(a)). Atomic force microscopy images (Fig. 1(b)) revealed a smooth surface morphology with a root mean square roughness of $0.6 \mathrm{~nm}$. P-type conductivity was confirmed in the as-grown $\mathrm{p}^{+} \mathrm{MoS}_{2} /$ sapphire film using Hall measurements with $\mathrm{Ni} / \mathrm{Au}$ contact pads e-beam evaporated at the four corners of the film. A sheet resistance of $164 \mathrm{k} \Omega / \square$, sheet charge density of $7.6 \times 10^{13} \mathrm{~cm}^{-2}$ and a Hall mobility of 0.5 $\mathrm{cm}^{2} / \mathrm{V}$ s was measured. The equivalent volume charge density is calculated to be $1.1 \times 10^{20} \mathrm{~cm}^{-3}$ from the measured sheet charge density and thickness of the film $(7 \mathrm{~nm})$. Ni/Au/Ni metal contacts were also patterned on large area $\mathrm{p}^{+} \mathrm{MoS}_{2}$ using optical lithography. Using a transfer 
length measurement structure (see Supplementary Figure S1), the resistance was measured as a function of length between contact pads ( 5 um to $25 \mathrm{um}$ ). From the linear fit of the total resistance versus length, a sheet resistance value of $154 \mathrm{k} \Omega / \square$ was extracted. The sheet resistance value extracted from transfer length measurement structure is in close agreement with the value extracted from large area hall measurements, indicating uniform conductivity across the large area $\mathrm{p}-\mathrm{MoS}_{2}$ sample.

$\mathrm{n}^{+} \mathrm{GaN}(20 \mathrm{~nm})$ was grown on $\mathrm{n}-\mathrm{GaN} / \mathrm{Sapphire}$ templates using $\mathrm{N}_{2}$ plasma-assisted molecular beam epitaxy (MBE) in Ga-rich growth conditions ${ }^{27}$ at a substrate temperature of $700^{\circ}$ $\mathrm{C}$ using standard effusion cells for gallium and silicon dopant. A high silicon doping density of 5 $\mathrm{x} 10^{19} \mathrm{~cm}^{-3}$ was used to enhance interband tunneling. The target doping density was achieved using SIMS measurements on calibration samples (not shown here). The Ga-rich growth conditions resulted in excess Ga metal droplets on the surface, which were removed using hydrochloric acid.

$\mathrm{p}^{+} \mathrm{MoS}_{2}$ was transferred onto MBE-grown $\mathrm{n}^{+} \mathrm{GaN}$ using a benign DI water-based process $^{28}$ reported earlier ${ }^{29}$. A PDMS stamp was pressed on to $\mathrm{p}^{+} \mathrm{MoS}_{2} /$ sapphire and detached to create an air gap at the interface. The sample was then immersed in DI water. The $\mathrm{MoS}_{2}$ layer was lifted off from the substrate and mm-sized fragments of $\mathrm{MoS}_{2}$ films floated in DI water. $\mathrm{p}^{+}$ $\mathrm{MoS}_{2}$ was then fished out using MBE-grown ${ }^{+}$GaN/sapphire sample. The sample was then dried overnight and annealed in a vacuum chamber at $110{ }^{\circ} \mathrm{C}$ to remove any water molecules trapped at the $\mathrm{MoS}_{2} / \mathrm{GaN}$ interface. The resulting device stack is shown in Fig. 2 (a). An optical microscope image of the layer-transferred $\mathrm{MoS}_{2} / \mathrm{GaN}$ diode is shown in Fig. 2 (b), showing the $\mathrm{Ni} / \mathrm{Au} / \mathrm{Ni}$ metal contacts on continuous $\mathrm{MoS}_{2}$ layers. Raman spectra (Renishaw, $514 \mathrm{~nm}$ laser) of as-grown $\mathrm{p}^{+} \mathrm{MoS}_{2} /$ sapphire, $\mathrm{n}^{+} \mathrm{GaN} / \mathrm{sapphire}$ and layer transferred $\mathrm{p}^{+} \mathrm{MoS}_{2} / \mathrm{n}^{+} \mathrm{GaN} /$ sapphire is 
shown in Fig. 2 (c). The characteristic $\mathrm{E}_{2 \mathrm{~g}}^{1}$ and $\mathrm{A}_{1 \mathrm{~g}}$ vibrational modes were observed with a peak separation of $26 \mathrm{~cm}^{-1}$ were observed and a peak intensity ratio of 1.16 in as-grown $\mathrm{p}^{+} \mathrm{MoS}_{2} /$ sapphire. Epitaxial GaN/sapphire showed a very strong Raman peak at $570 \mathrm{~cm}^{-1}$ corresponding to the $\mathrm{E}_{2}$ mode in $\mathrm{GaN}$ and a faint $\mathrm{A}_{1 \mathrm{~g}}$ mode $\left(419 \mathrm{~cm}^{-1}\right)$ corresponding to c-plane sapphire. Both the characteristic $\mathrm{E}_{2 \mathrm{~g}}^{1}$ and $\mathrm{A}_{1 \mathrm{~g}}$ modes of $\mathrm{MoS}_{2}$ (peak ratio of 1.08) as well as the $\mathrm{E}_{2}$ mode of $\mathrm{GaN}$ were observed in the layer-transferred $\mathrm{MoS}_{2} / \mathrm{GaN}$ sample. However, the signal to noise ratio in the $\mathrm{MoS}_{2} / \mathrm{GaN}$ was about one third of the as-grown $\mathrm{MoS}_{2} /$ sapphire indicating degradation of material quality during layer transfer. Inductively coupled plasma/reactive ion etching (ICP-RIE) with $\mathrm{BCl}_{3} / \mathrm{Ar}$-based chemistry was used to etch $\mathrm{MoS}_{2}$ using $\mathrm{Ni} / \mathrm{Au} / \mathrm{Ni}$ metal stack as the etch mask for device isolation. Large area $\mathrm{Ni} / \mathrm{Au} / \mathrm{Ni}$ metal pads directly in contact with $\mathrm{n}^{+} \mathrm{GaN}$ (regions with no $\mathrm{MoS}_{2}$ ) was used as ohmic contacts to $\mathrm{n}^{+} \mathrm{GaN}$.

A schematic of the equilibrium band diagram of the $\mathrm{p}^{+} \mathrm{MoS}_{2} / \mathrm{n}^{+} \mathrm{GaN}$ tunnel diode is shown in Fig. 2(d). Here, the experimentally measured conduction band offset $(\sim 0.2 \mathrm{eV})$ between $\mathrm{MoS}_{2}$ and GaN from a previous report is used ${ }^{10}$. It should be noted that there is a van der Waals gap at the $\mathrm{MoS}_{2} / \mathrm{GaN}$ interface and a van der Waals gap between each S-Mo-S layers of $\mathrm{MoS}_{2}$. The qualitative charge profile in the $\mathrm{MoS}_{2} / \mathrm{GaN}$ TJ is also shown in Figure 2(d). The space charge regions due to depletion of donors in $\mathrm{GaN}$, depletion of acceptors in $\mathrm{MoS}_{2}$, negative spontaneous polarization ${ }^{30}$ sheet charge at the surface of $\mathrm{GaN}$, charged surface donors ${ }^{31}$ and unintentional positive sheet charge due to $\mathrm{O}, \mathrm{Si}$ contaminants ${ }^{32}$ at the GaN surface play an important role in the electrostatics of the $\mathrm{MoS}_{2} / \mathrm{GaN}$ junction. In addition to the sources of charge mentioned above, the $\mathrm{MoS}_{2} / \mathrm{GaN}$ band offset is expected to be different from the electron affinity difference due to the presence of interfacial dipoles ${ }^{33}$. Heavy doping on both sides of the junction can considerably reduce the depletion region width. The total positive sheet charge due 
to surface states and $\mathrm{O}, \mathrm{Si}$ contaminants is denoted as $\mathrm{N}_{\text {interface, net }}{ }^{+}$. Under forward bias (Fig. 2 $(\mathrm{e})$ ), electrons in the conduction band ( $\Gamma$ valley) of GaN tunnel into empty states (holes) available in the valence band of multilayer $\operatorname{MoS}_{2}$ ( $\Gamma$ valley). Although multilayer $\mathrm{MoS}_{2}$ is an indirect gap semiconductor, the interband tunneling process between the $\Gamma$ valleys is expected to be akin to a direct tunneling process. With increasing forward bias, the tunneling current is expected to increase until the conduction and valence band are out of alignment. Beyond this forward bias, the tunneling current drop until forward bias diffusion current becomes the dominant conduction mechanism. This results in negative differential resistance under forward bias. Under reverse bias (Fig. 2(f)), the electrons in the valence band of $\mathrm{MoS}_{2}$ tunnel into empty states in the conduction band of GaN, and this is referred to as Zener tunneling.

Z-contrast scanning transmission electron microscope (STEM) images of GaN/MoS 2 tunnel diode device is shown in Fig.3. Individual 2D layers of $\mathrm{MoS}_{2}$, the van der Waals gaps, and the individual Mo and S atoms could be resolved in the STEM images. In comparison to previous reports of oxidized interfaces between $2 \mathrm{D}$ material and bulk semiconductors ${ }^{19}$, the heterojunction between layer-transferred $\mathrm{MoS}_{2}$ and $\mathrm{GaN}$ reported in this work is fairly abrupt in nature. The basal plane spacing ('a' lattice spacing) measured from the STEM images indicated alignment of the basal planes of GaN and $\mathrm{MoS}_{2}$.

Electrical characteristics of $\mathrm{p}^{+} \mathrm{MoS}_{2} / \mathrm{n}^{+} \mathrm{GaN}$ tunnel diodes are shown in Fig. 4. Under reverse bias, a very high current density was measured even in low reverse bias regime. A reverse current density of $1 \mathrm{kA} / \mathrm{cm}^{2}\left(315 \mathrm{~A} / \mathrm{cm}^{2}\right)$ was measured at a reverse bias of $1 \mathrm{~V}(0.5 \mathrm{~V})$. Under forward bias, negative differential resistance was observed at room temperature, which is a characteristic feature of interband tunneling in Esaki diodes. A peak current density of 446 $\mathrm{A} / \mathrm{cm}^{2}$ was measured at a forward bias voltage of $0.8 \mathrm{~V}$. A valley current of $368 \mathrm{~A} / \mathrm{cm}^{2}$ was 
measured at a voltage of $1.2 \mathrm{~V}$, with a peak to valley current ratio of 1.2. Negative differential resistance was found to be repeatable and robust during multiple cycles of measurements. The double hump observed in the negative differential resistance regime (inset to Fig. 4 (b)) is attributed to the oscillations in the measurement circuit due to the presence of negative resistance and is a measurement $\operatorname{artifact}^{34}$.

The device exhibited minimal hysteresis in its IV characteristics when the voltage sweep direction was reversed (Fig.4 (c)). The minimal hysteresis observed in a layer-transferred, nonepitaxial heterojunction such as this indicates the possibility of making high quality 2D/3D heterojunctions with minimal charge trapping for a wide range of device applications.

SILVACO ATLAS ${ }^{35}$ was used to model $\mathrm{p}^{+} \mathrm{MoS}_{2} / \mathrm{GaN}$ TJ. Multilayer $\mathrm{MoS}_{2}$ was treated as a bulk 3D semiconductor for simulation purposes, and a conduction band offset of $0.2 \mathrm{eV}$ was used based on previous experimental results from $\mathrm{MoS}_{2} / \mathrm{GaN}$ heterojunctions ${ }^{10}$. A hole effective mass of $0.5996 \mathrm{~m}_{0}^{36}$ was used. Holes are populated in the $\Gamma$ valley of the valence band in $\mathrm{p}^{+}$ $\mathrm{MoS}_{2}$, and hence the tunneling process is expected to be direct. As such, the direct band gap value of $\mathrm{MoS}_{2}(1.2 \mathrm{eV})$ was used for the simulation. The following parameters were used in the simulation: Conduction band effective density of states (DOS) ${ }^{35}$ of $6.222 \mathrm{e}+018 \mathrm{~cm}^{-3}$, Valence band effective DOS of $6.516 \mathrm{e}+018 \mathrm{~cm}^{-3}$ and Electron affinity ${ }^{37}$ of $3.8 \mathrm{eV}$. Band structure was computed in SILVACO using k.p method and tunneling current was computed using non-local band to band tunneling model.

Tunneling current density was computed at room temperature for different values of

$\mathrm{N}_{\text {interface, net }}{ }^{+}$. The peak current density calculated in the case of $\mathrm{N}_{\text {interface, net }}{ }^{+}=4 \times 10^{13} \mathrm{~cm}^{-2}$ matches the experimentally observed peak current density (denoted as star in Figures 5 (c)). It 
should be noted that the calculated current density is "intrinsic" tunneling current. In reality, series and contact resistances increases the total voltage drop in the device. The total voltage drop in the device can be computed as $\mathrm{V}_{\text {EXPT }}=\mathrm{V}_{\text {SIMULATON,TJ }}+\mathrm{J}_{\text {SIMULATION,TJ }} * \rho_{\text {series. }}$ Using $\rho_{\text {series }}=1.7 \times 10^{-3} \Omega \mathrm{cm}^{2}$, and the computed I-V curve for $\mathrm{N}_{\text {interface, net }}{ }^{+}=4 \times 10^{13} \mathrm{~cm}^{-2}$, the model described here was successfully able to match the experimental peak voltage, forward and reverse bias current density (Fig. 5 (d)). This indicates that the electrostatics at the 2D/3D heterojunction, interface sheet charge and polarization charge in this case, play a crucial role in determining the electrical characteristics of the 2D/3D heterojunction.

The low resistance $\mathrm{GaN} / \mathrm{MoS}_{2}$ tunnel diodes reported here can enable a number of novel device applications. With controlled p-type doping, n-type doping and gating, steep slope tunneling FETs can be realized with $\mathrm{GaN} / \mathrm{MoS}_{2}$ tunnel diodes. The 2D/3D tunnel junction reported here, in conjunction with 2D/2D tunnel junctions can enable series connection of multiple atomically thin 2D heterostructure-based active regions for applications such as multi junction solar cells, multiple color light emitting diodes and multi-spectral photodetectors. With no constraints of lattice matching, vertical heterostructures with extreme band gaps can be integrated and connected in series with such tunnel junctions. Traditionally interband tunnel junctions have been realized in epitaxial heterostructures. This work demonstrates the potential of non-epitaxial heterostructures for high performance device applications.

In summary, we report high current density 2D/3D tunnel junctions with non-epitaxial layer-transferred $\mathrm{p}+\mathrm{MoS}_{2}$ on to $\mathrm{n}^{+} \mathrm{GaN}$. Repeatable, room temperature negative differential resistance with a peak current of $446 \mathrm{~A} / \mathrm{cm}^{2}$, peak to valley ratio of 1.2 and minimal hysteresis is reported. Such 2D/3D heterojunction-based tunnel diodes can enable novel high performance vertical heterostructures based on 2D-layered materials and bulk semiconductors. 


\section{FIGURES}

Table of Contents Figure
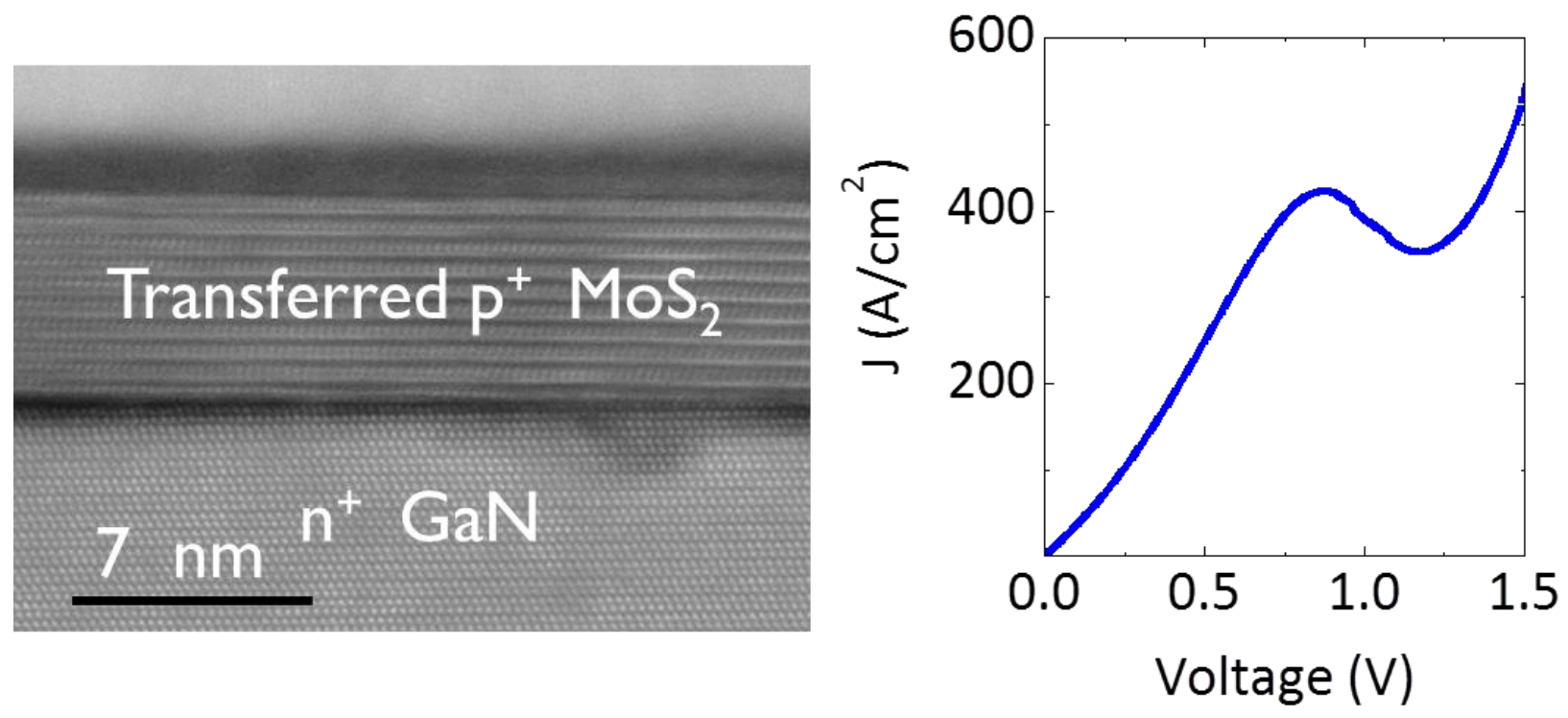


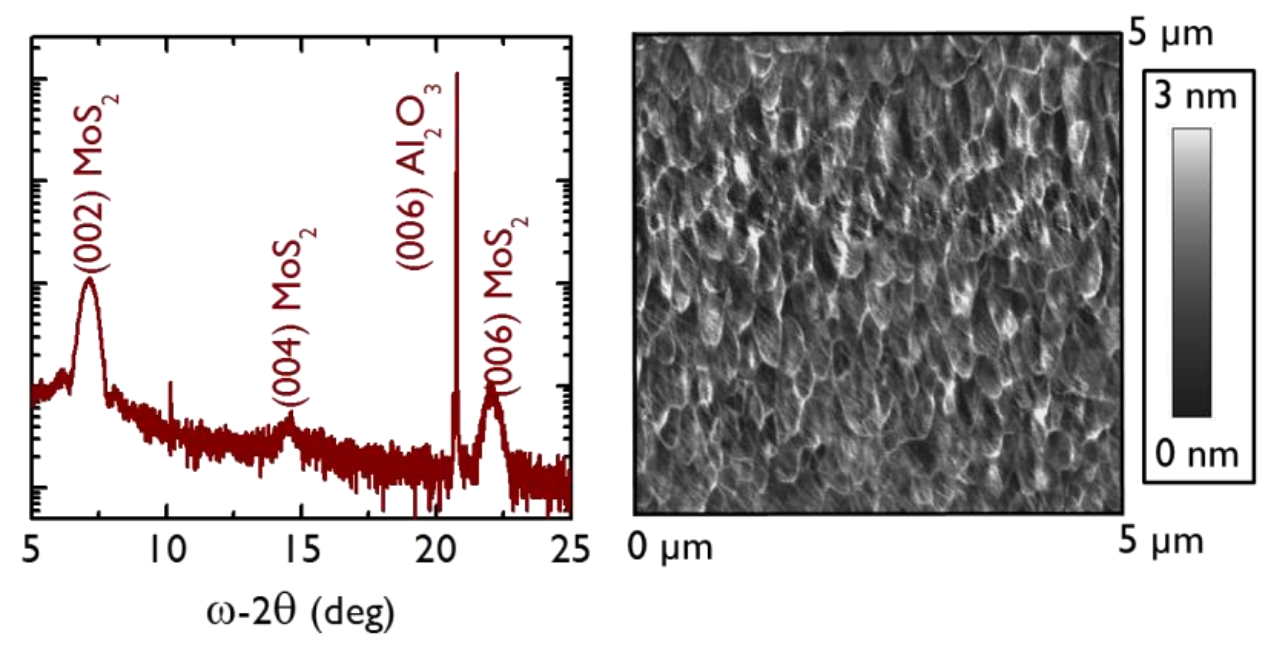

Figure 1. (a) X-ray diffraction data of crystalline large area $\mathrm{p}^{+} \mathrm{MoS}_{2}$ grown by chalcogenization of sputtered $\mathrm{Mo} / \mathrm{Nb} / \mathrm{Mo}$ stack on sapphire substrates (b) Atomic force microscope image of $\mathrm{p}^{+}$ $\mathrm{MoS}_{2}$ indicating smooth surface morphology (RMS roughness $\left.=0.6 \mathrm{~nm}\right)$. 


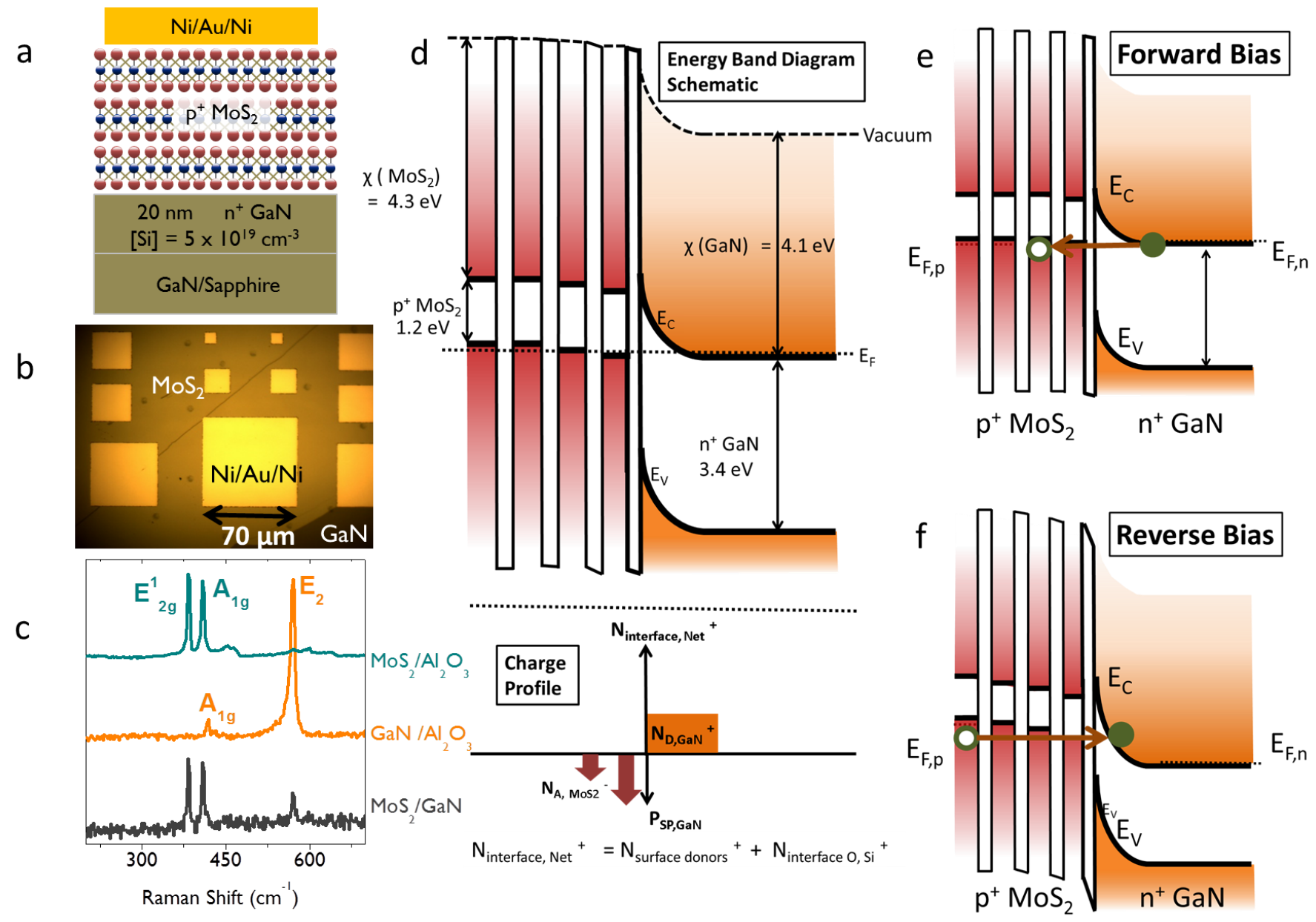

Figure 2. (a) Schematic of $\mathrm{p}^{+} \mathrm{MoS}_{2} / \mathrm{n}^{+}$GaN device structure, (b) Optical micrograph image of $\mathrm{MoS}_{2} / \mathrm{GaN}$ tunnel diode with $\mathrm{Ni} / \mathrm{Au} / \mathrm{Ni}$ ohmic contact to $\mathrm{p}^{+} \mathrm{MoS}_{2}$. Ni/Au/Ni is used as a etch mask to etch $\mathrm{MoS}_{2}$ to realize device isolation. (c) Raman spectra of as-grown $\mathrm{MoS}_{2} / \mathrm{sapphire}$, MBE-grown $\mathrm{n}^{+}$GaN/sapphire and layer-transferred $\mathrm{MoS}_{2} / \mathrm{GaN}$ tunnel diode showing characteristic Raman peaks. (d) Equilibrium energy band diagram and charge diagram schematic showing various components of charge contribution to the junction electrostatics. (e) Schematic band diagram under forward bias and (f) reverse bias illustrating the interband tunneling process. 


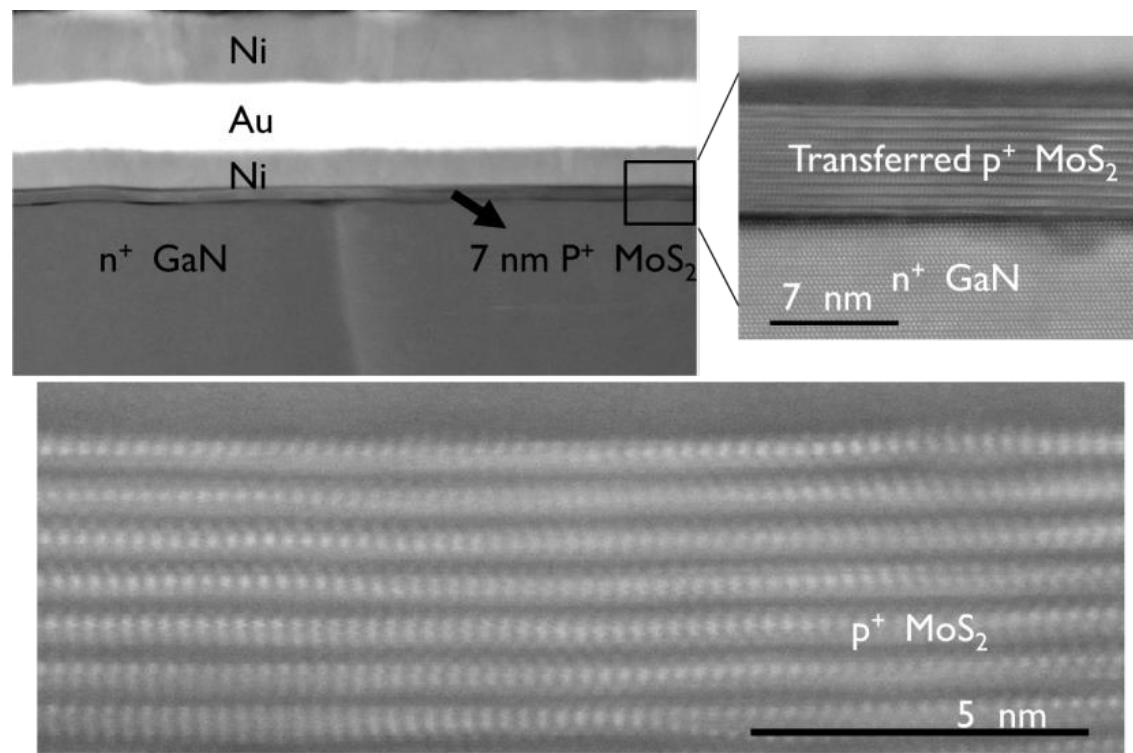

Figure 3. Scanning transmission electron microscope images of layer transferred $\mathrm{p}^{+} \mathrm{MoS}_{2} / \mathrm{n}^{+}$ GaN tunnel diode device showing continuous $\mathrm{MoS}_{2}$ coverage, abrupt interface, individual Mo, $\mathrm{S}$ atoms and van der Waals gaps.
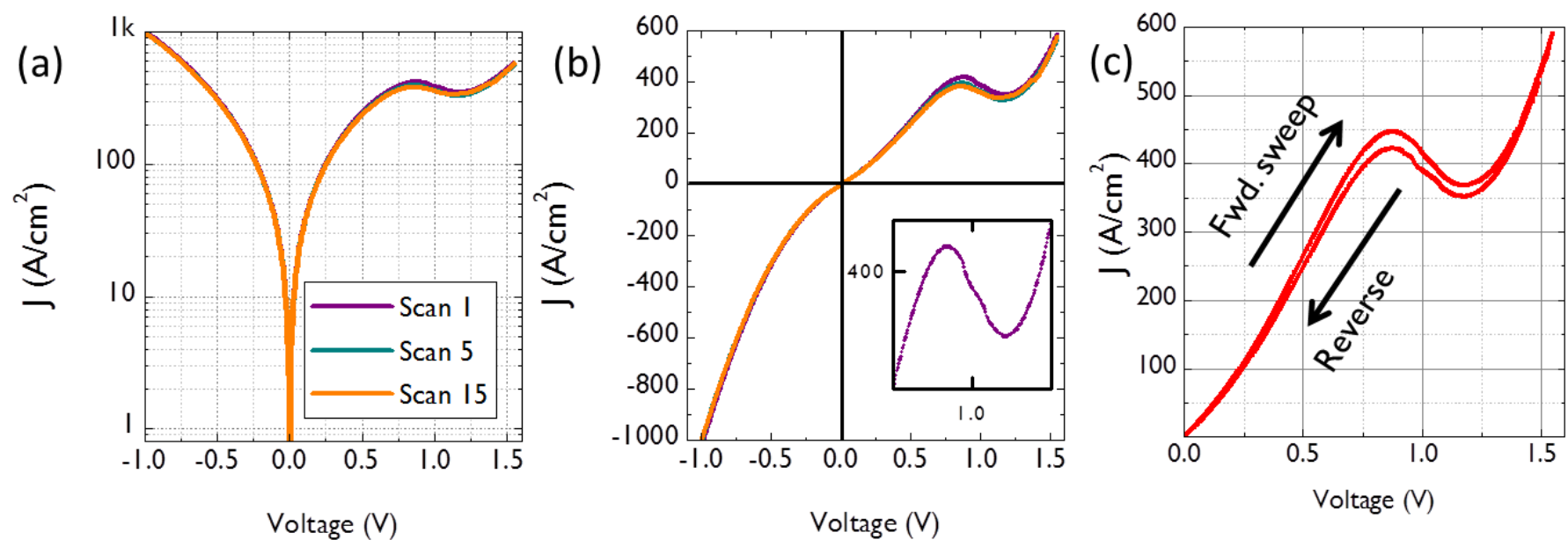

Figure 4. (a) Linear, (b) log current-voltage characteristics of $\mathrm{p}^{+} \mathrm{MoS}_{2} / \mathrm{n}^{+} \mathrm{GaN}$ tunnel diode showing repeatable room temperature negative differential resistance, and (c) minimal hysteresis measured in between forward and reverse sweeps. 

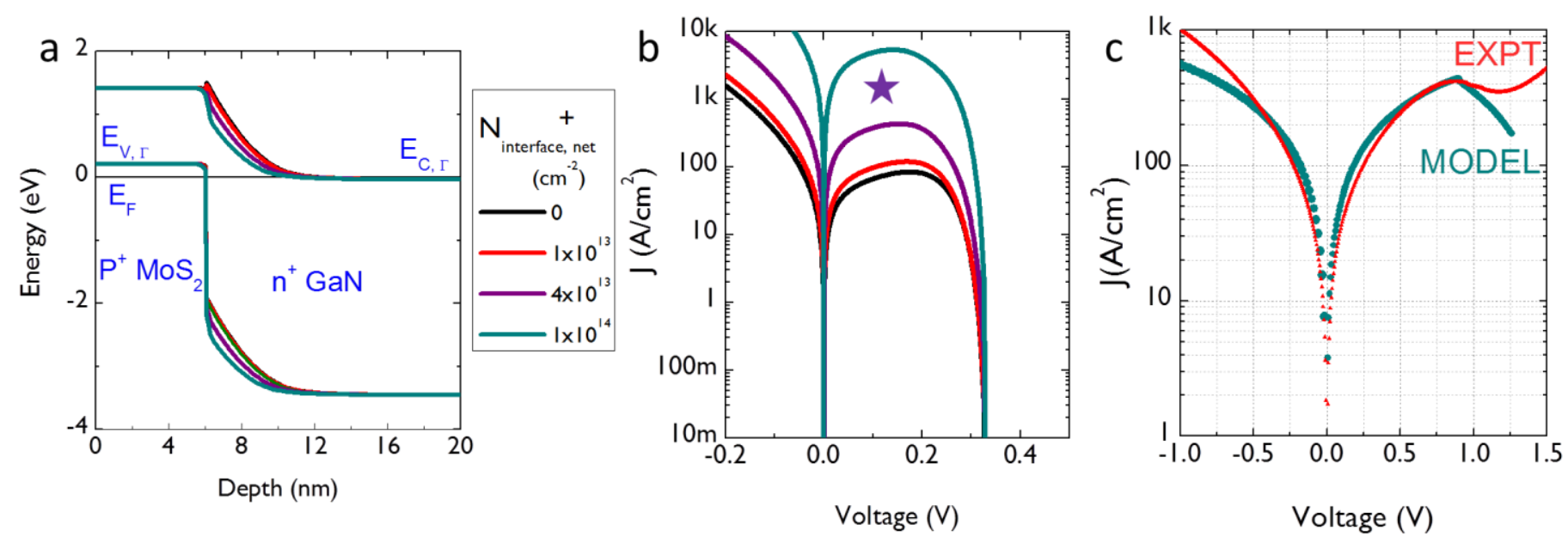

Figure 5. (a) Equilibrium band diagram, (b) Log J-V characteristics of the "intrinsic" tunnel diode as a function of net interface positive charge $\left(\mathrm{N}_{\text {interface,net }}{ }^{+}\right)$. The calculated peak current density value matches with the experimentally measured value for $\mathrm{N}_{\text {interface, net }}{ }^{+}=4 \mathrm{E} 13 \mathrm{~cm}^{-2}$. (d) With the addition of series resistance, the simulated IV characterisitcs match with the experimentally measured characteristics.

\section{ASSOCIATED CONTENT}

Supporting Information. Discussion of sheet resistance measurement in $\mathrm{p}^{+} \mathrm{MoS}_{2}$, Surface morphology of MBE-grown GaN/Sapphire, layer transferred $\mathrm{MoS}_{2}$ on GaN, Effect of n-type doping in $\mathrm{MoS}_{2} / \mathrm{GaN}$ TJ. This material is available free of charge via the Internet at http://pubs.acs.org.

\section{AUTHOR INFORMATION}

\section{Corresponding Author}

*Email:krishnamoorthy.13@osu.edu; rajan@ece.osu.edu

\section{ACKNOWLEDGMENT}


The authors would like to acknowledge the support from Air Force Office of Scientific

Research (Program manager: Dr. Kenneth Goretta) under Contract No. FA9550-15-1-0294.

\section{REFERENCES}

(1) Radisavljevic, B.; Radenovic, A.; Brivio, J.; Giacometti, V.; Kis, A. Single-layer $\mathrm{MoS}_{2}$ transistors. Nature nanotechnology 2011, 6(3), 147-150.

(2) Esmaeili-Rad, Mohammad R., and Sayeef Salahuddin. "High performance molybdenum disulfide amorphous silicon heterojunction photodetector." Scientific reports 3 (2013).

(3) Wang, Liu, Jiansheng Jie, Zhibin Shao, Qing Zhang, Xiaohong Zhang, Yuming Wang, Zheng Sun, and Shuit-Tong Lee. "MoS2/Si Heterojunction with Vertically Standing Layered Structure for Ultrafast, High-Detectivity, Self-Driven Visible-Near Infrared Photodetectors." Advanced Functional Materials 25, no. 19 (2015): 2910-2919.

(4) Li, Bo, Gang Shi, Sidong Lei, Yongmin He, Weilu Gao, Yongji Gong, Gonglan Ye et al. "3D Band Diagram and Photoexcitation of 2D-3D Semiconductor Heterojunctions." Nano letters 15, no. 9 (2015): 5919-5925.

(5) Hao, Lanzhong, Yunjie Liu, Wei Gao, Zhide Han, Qingzhong Xue, Huizhong Zeng, Zhipeng $\mathrm{Wu}$, Jun Zhu, and Wanli Zhang. "Electrical and photovoltaic characteristics of MoS2/Si pn junctions." Journal of Applied Physics 117, no. 11 (2015): 114502.

(6) Lin, Shisheng, Xiaoqiang Li, Peng Wang, Zhijuan Xu, Shengjiao Zhang, Huikai Zhong, Zhiqian $\mathrm{Wu}$, Wenli $\mathrm{Xu}$, and Hongsheng Chen. "Interface designed MoS2/GaAs heterostructure solar cell with sandwich stacked hexagonal boron nitride." Scientific reports 5 (2015).

(7) Xu, Zhijuan, Shisheng Lin, Xiaoqiang Li, Shengjiao Zhang, Zhiqian Wu, Wenli Xu, Yanghua $\mathrm{Lu}$, and Sen $\mathrm{Xu}$. "Monolayer MoS2/GaAs heterostructure self-driven photodetector with extremely high detectivity." arXiv preprint arXiv:1512.06867 (2015).

(8) Lin, Shisheng, Peng Wang, Xiaoqiang Li, Zhiqian Wu, Zhijuan Xu, Shengjiao Zhang, and Wenli Xu. "Gate tunable monolayer MoS2/InP heterostructure solar cells." Applied Physics Letters 107, no. 15 (2015): 153904.

(9) Lee II, Edwin W., Lu Ma, Digbijoy N. Nath, Choong Hee Lee, Aaron Arehart, Yiying Wu, and Siddharth Rajan. "Growth and electrical characterization of two-dimensional layered MoS2/SiC heterojunctions." Applied Physics Letters 105, no. 20 (2014): 203504. 
(10) Lee II, Edwin W., Choong Hee Lee, Pran K. Paul, Lu Ma, William D. McCulloch, Sriram Krishnamoorthy, Yiying Wu, Aaron R. Arehart, and Siddharth Rajan. "Layer-transferred MoS2/GaN PN diodes." Applied Physics Letters 2015, 107, 10,103505.

(11) Ruzmetov, Dmitry, Kehao Zhang, Gheorghe Stan, Berc Kalanyan, Ganesh R. Bhimanapati, Sarah M. Eichfeld, Robert A. Burke et al. "Vertical 2D/3D Semiconductor Heterostructures Based on Epitaxial Molybdenum Disulfide and Gallium Nitride." ACS nano 2016.

(12) Liao, Jiamin, Baisheng Sa, Jian Zhou, Rajeev Ahuja, and Zhimei Sun. "Design of highefficiency visible-light photocatalysts for water splitting: MoS2/AlN ( $\mathrm{GaN})$ heterostructures." The Journal of Physical Chemistry C 118, no. 31 (2014): 17594-17599.

(13) Jeong, Hyun, Seungho Bang, Hye Min Oh, Hyeon Jun Jeong, Sung-Jin An, Gang Hee Han, Hyun Kim et al. "Semiconductor-Insulator-Semiconductor Diode Consisting of Monolayer MoS2, h-BN, and GaN Heterostructure." ACS nano 9, no. 10 (2015): 10032-10038.

(14) Lee, C.H.; Lee, G.H.; Van Der Zande, A.M.; Chen, W.; Li, Y.; Han, M.; Cui, X.; Arefe, G.; Nuckolls, C.; Heinz, T.F.; Guo, J. ; Hone, J.; Kim, P. Atomically thin p-n junctions with van der Waals heterointerfaces. Nature nanotechnology 2014, 9(9), pp.676-681.

(15) Roy, T.; Tosun, M.; Cao, X.; Fang, H.; Lien, D. H.; Zhao, P.; Chen, Y.Z.; Chueh, Y.L.; Guo, J; Javey, A. Dual-Gated MoS2/WSe2 van der waals tunnel diodes and transistors. ACS Nano 2015, 9(2), 2071-2079.

(16) Nourbakhsh, A.; Zubair, A.; Dresselhaus, M.S.; Palacios, T. Transport Properties of a $\mathrm{MoS}_{2} / \mathrm{WSe}_{2}$ Heterojunction Transistor and its Potential for Application. Nano letters 2016, 16 (2), pp 1359-1366

(17) Yan, R.; Fathipour, S.; Han, Y.; Song, B.; Xiao, S.; Li, M.; Ma, N.; Protasenko, V.; Muller, D.A.; Jena, D. ; Xing, H.G. Esaki diodes in van der Waals heterojunctions with broken-gap energy band alignment. Nano letters 2015, 15(9), pp.5791-5798.

(18) Roy, T.; Tosun, M.; Hettick, M.; Ahn, G.H.; Hu, C. ; Javey, A. 2D-2D tunneling field-effect transistors using $\mathrm{WSe}_{2} / \mathrm{SnSe}_{2}$ heterostructures. Applied Physics Letters 2016, 108(8), p.083111.

(19) Sarkar, D.; Xie, X.; Liu, W.; Cao, W.; Kang, J.; Gong, Y.; Kraemer, S.; Ajayan, P.M.; Banerjee, K. A subthermionic tunnel field-effect transistor with an atomically thin channel. Nature 2015, 526(7571), pp.91-95.

(20) Tanaka, T., A. Watanabe, H. Amano, Y. Kobayashi, I. Akasaki, S. Yamazaki, and M. Koike. "p-type conduction in Mg-doped $\mathrm{GaN}$ and Al0. $08 \mathrm{Ga} 0.92 \mathrm{~N}$ grown by metalorganic vapor phase epitaxy." Applied physics letters 65, no. 5 (1994): 593-594. 
(21) Park, C. H., S. B. Zhang, and Su-Huai Wei. "Origin of p-type doping difficulty in ZnO: The impurity perspective." Physical Review B 66, no. 7 (2002): 073202.

(22) Esaki, L. New phenomenon in narrow germanium $p-n$ junctions. Physical review 1958, $109(2)$, p.603.

(23) J. A. Wilson and A. D. Yoffe, Adv. Phys. 18(73), 193-335 (1969)

(24) R. S. Title and M. W. Shafer, Phys. Rev. Lett. 28(13), 808-810 (1972)

(25) Ma, L.; Nath , D.N.; Lee II ,E.W.; Lee, C.H.; Yu,M.; Arehart, A.; Rajan, S.; Wu, Y. Epitaxial growth of large area single-crystalline few-layer MoS2 with high space charge mobility of $192 \mathrm{~cm}^{2} \mathrm{~V}^{-1} \mathrm{~s}^{-1}$." Applied Physics Letters 105, no. 7 (2014): 072105.

(26) Laskar, Masihhur R., Digbijoy N. Nath, Lu Ma, Edwin W. Lee II, Choong Hee Lee, Thomas Kent, Zihao Yang et al. "p-type doping of MoS2 thin films using Nb." Applied Physics Letters 104, no. 9 (2014) : 092104.

(27) Tarsa, E. J., B. Heying, X. H. Wu, P. Fini, S. P. DenBaars, and J. S. Speck. "Homoepitaxial growth of GaN under Ga-stable and $\mathrm{N}$-stable conditions by plasma-assisted molecular beam epitaxy." Journal of applied physics 82, no. 11 (1997): 5472-5479.

(28) Gurarslan, A.; Yu, Y.; Su, L.; Yu, Y.; Suarez, F.; Yao, S.; Zhu, Y.; Ozturk, M.; Zhang, Y.; Cao, L. Surface-energy-assisted perfect transfer of centimeter-scale monolayer and few-layer MoS2 films onto arbitrary substrates. ACS nano 2014, 8(11), pp.11522-11528.

(29) Lee, Choong Hee, William McCulloch, Edwin W. Lee II, Lu Ma, Sriram Krishnamoorthy, Jinwoo Hwang, Yiying Wu, and Siddharth Rajan. "Transferred large area single crystal MoS2 field effect transistors." Applied Physics Letters 107, no. 19 (2015): 193503.

(30) Bernardini, F.; Fiorentini, V. ; Vanderbilt, D. Spontaneous polarization and piezoelectric constants of III-V nitrides. Physical Review B 1997, 56(16), p.R10024.

(31) Ibbetson, J.P.; Fini, P.T.; Ness, K.D.; DenBaars, S.P.; Speck, J.S.; Mishra, U.K. Polarization effects, surface states, and the source of electrons in AlGaN/GaN heterostructure field effect transistors. Applied Physics Letters 2000, 77(2), pp.250-252.

(32) Lee, W.; Ryou, J.H.; Yoo, D.; Limb, J.; Dupuis, R.D.; Hanser, D.; Preble, E.; Williams, N.M. ; Evans, K. Optimization of Fe doping at the regrowth interface of GaN for applications to 
III-nitride-based heterostructure field-effect transistors. Applied Physics Letters 2007, 90(9), p.093509.

(33) Schlaf, R.; Lang, O.; Pettenkofer, C.; Jaegermann, W. Band lineup of layered semiconductor heterointerfaces prepared by van der Waals epitaxy: Charge transfer correction term for the electron affinity rule. Journal of applied physics 1999, 85(5), pp.2732-2753.

(34) Bao, M.; Wang, K.L. Accurately measuring current-voltage characteristics of tunnel diodes. IEEE Transactions on Electron Devices, 2006, 53(10), pp.2564-2568.

(35) http://www.silvaco.com/products/tcad/device_simulation/atlas/atlas.html

(36) Liu, L., Kumar, S.B., Ouyang, Y. and Guo, J., 2011. Performance limits of monolayer transition metal dichalcogenide transistors. Electron Devices, IEEE Transactions on, 58(9), pp.3042-3047.

(37) Lee, K., Kim, H.Y., Lotya, M., Coleman, J.N., Kim, G.T. and Duesberg, G.S., 2011. Electrical characteristics of molybdenum disulfide flakes produced by liquid exfoliation. Advanced Materials, 23(36), pp.4178-4182. 